\title{
Characterization of floc size, strength and structure under various coagulation mechanisms
}

\author{
Tao $\mathrm{Li}^{\mathrm{a}}$, Zhe Zhu ${ }^{\mathrm{a}, \mathrm{b}}$, Dongsheng Wang ${ }^{\mathrm{a}, *}$, Chonghua Yao ${ }^{\mathrm{b}}$, Hongxiao Tang ${ }^{\mathrm{a}}$ \\ ${ }^{a}$ State Key Laboratory of Environmental Aquatic Chemistry, Research Center for Eco-Environmental Sciences, Chinese Academy of Sciences, \\ Beijing 100085, PR China \\ ${ }^{\mathrm{b}}$ Resources and Environmental Engineering Institute, East China University of Science and Technology, Shanghai 200237, PR China
}

Received 28 April 2006; received in revised form 5 July 2006; accepted 6 July 2006

Available online 13 July 2006

\begin{abstract}
Flocs generated by various coagulation mechanisms exhibit different size, strength and structure. The properties and fractal dimensions of flocs formed under three common coagulation mechanisms, i.e. charge neutralization, sweep and bridging, were investigated at various hydraulic conditions. The results showed that the floc size decreased with the increasing average velocity gradient $G$ and the stable floc size exponent $\gamma$ was of the following hierarchy: charge neutralization $(0.6107)>$ sweep $(0.5618)>$ bridging $(0.3674)$. Furthermore, fractal dimensions of flocs were the highest when formed by sweep and the lowest when generated by bridging flocculation. The mass fractal dimensions measured by light scattering were between 2.0 and 3.0 and the floc strength was between 0.01 and $0.58 \mathrm{~N} \mathrm{~m}^{-2}$. An intrinsic unity of the relationship among floc size, fractal dimensions, floc strength under the three coagulation mechanisms was demonstrated.
\end{abstract}

(C) 2006 Elsevier B.V. All rights reserved.

Keywords: Floc; Size; Fractal dimension; Strength; Coagulation mechanism

\section{Introduction}

\subsection{Coagulation mechanisms}

Coagulation is a well-established process in water treatment to remove suspended particles by combining small particles into larger aggregates. Three mechanisms, i.e. charge neutralization, sweeping and bridging have been demonstrated to exist during coagulation. Coagulants based on hydrolyzing metal salts are widely used. The metal salts hydrolyze rapidly to form various cationic species, which are adsorbed by negatively charged particles and cause charge reduction. At low coagulant dosages, charge neutralization is a possible mechanism of particle destabilization [1]. On the other hand, when a metal salt is added to water at a concentration sufficiently high to cause precipitation of amorphous metal hydroxide, colloidal particles can be enmeshed in these precipitates. This is called "sweep flocculation" [2]. Moreover, destabilization by bridging occurs when segments of a polymer chain absorb on more than one

\footnotetext{
* Corresponding author. Tel.: +86 10 62849138; fax: +86 1062923543 .

E-mail address: wgds@rcees.ac.cn (D. Wang).
}

particle, thereby linking the particles together. One obvious consequence of bridging flocculation is that the flocs produced can be much stronger than those formed when particles are destabilized by simple salts $[3,4]$, and thus, aggregates formed by polymeric flocculants appear to be significantly more resistant to breakage [5].

\subsection{Fractal dimension}

It has been well-known that aggregates formed during the coagulation process exhibit a fractal characteristic, implying that they are self-similar and scale invariant [6-9]. Fractal theories for particle aggregates provide a new quantitative method to describe the structure of particles aggregates in various water systems [10]. The essentially useful feature of fractal geometry is the recognition of scale invariance as a measure to characterize structures. For an aggregate, the relationship between mass $(M)$ and size $(R)$ is

$M \propto R^{D_{F}}$

where $D_{\mathrm{F}}$ is the mass fractal dimension and has a value between 1 and 3 in three-dimensional space [11]. Densely 
packed aggregates have a high fractal dimension, while lower fractal dimension results from large, highly branched and loosely bound structures. At present, three common techniques for fractal dimension measurement have been developed, i.e. light scattering, settling and image analysis [12-15].

\subsection{Floc strength}

In addition to size, density and structure are the aggregate properties commonly investigated in solid-liquid separation; floc strength is the other particularly important operational parameter that deserves special attention [16]. However, floc strength is dependent upon the bonds between the components of the aggregate. The floc structure and particle bond strength, as well as number of individual bonds, are interrelated with floc strength [17]. Therefore, when the stress on floc surface is larger than the bonding strength, a floc will be broken [18]. However floc strength is harder to characterize and there is, as yet, no straightforward technique to characterize the floc strength experimentally. Generally, there are two modes of floc rupture [18], surface erosion and large-scale fragmentation $[19,20]$. Researchers [20-22] found a correlation between floc size and the strength for a given rate of shear condition. Spicer and Pratsinis [23] studied on the flocculation of polystyrene particles in a stirred tank and pointed out that the average floc structure became less open or irregular as the floc size distribution attained steady state as a result of shear-induced breakage or restructuring.

Based on the relationship between the velocity gradient in the flocculating vessel and aggregates size, the relationship between stable floc size and the applied shear has been developed as an empirical expression:

$d=C G^{-\gamma}$

Linearisation of the equation allows values of $\gamma$ and $\log C$ to be found from the a $\log -\log$ plot of floc size measurement against the average velocity gradients [19]:

$\log d=\log C-\gamma \log G$

where $d$ is the floc diameter; $C$ is the floc strength constant that strongly depends on the method used for particle size measurement; $G$ is the average velocity gradient and $\gamma$ is the stable floc size exponent dependent upon floc break-up mode and the size of eddies that causes the breakage. Moreover, the steeper the slope $\gamma$, the more prone the flocs are to break into smaller sizes with increasing shear force, and thus, the $\gamma$ value is considered as an indicator of floc strength. Bache and Rasool [24] gave the value of slope, which varied between 0.44 and 0.64 , for alumino-humic flocs under low alkalinity conditions. Francois [20] concluded that the values of $\gamma$ were around 0.5 for all types of floc formed by alumino-kaolin under different aluminum sulphate dosage.

Spicer et al. [25] investigated the effect of shear history on the evolution of polystyrene-alum floc size, density and structure by small angle light scattering, the floc structure was characterized by the mass fractal dimension, $D_{\mathrm{F}}$, and the relative floc density.
Jung et al. [26] also used the small angle light scattering to investigate the formation of iron hydroxide flocs from the hydrolysis of $\mathrm{FeCl}_{3}$ solution and found that the freshly formed iron hydroxide flocs had a fractal dimension of $2.25 \pm 0.05$, and as the hydrolysis proceeded the floc grew and restructured to a more compact form with a fractal dimension of a $2.52 \pm 0.05$.

Although a number of methods have been developed for the floc strength measurement, there are no straightforward techniques to experimentally characterize the floc strength without destroying the flocs to some extent and there is limited literature for investigating the floc structure and floc strength under different coagulation mechanisms. The main objective of this investigation was to examine the morphological characteristics and strength of flocs formed under three common coagulation mechanisms, and to understand the effect of different coagulation mechanism on flocs structure and strength. Jar tests were conducted with focus on the relation between fractal dimensions and strength of flocs.

\section{Methods and materials}

\subsection{Suspension}

Kaolin was applied as a model suspension. The stock suspension of Kaolin was prepared in deionized water, which was similar to Yukselen and Gregory [4]. The solid concentration of suspension was determined by gravimetrically to be $137 \mathrm{~g}^{-1}$. The average size of the particles in suspension was close to $5 \mu \mathrm{m}$, measured by a laser diffraction instrument (Mastersize 2000, Malvern, UK).

\subsection{Coagulants}

Aluminum sulfate $\left(\mathrm{Al}_{2}\left(\mathrm{SO}_{4}\right)_{3} \cdot 18 \mathrm{H}_{2} \mathrm{O}\right.$, analytic reagent) was used as "alum" coagulant. 1.0 $\mathrm{M}$ stock alum solutions were prepared with deionized water.

A commercial cationic polyacrylamide (PAM) (Superfloc C498, Cytec Industries, USA) was used as the flocculant, which has a molecular weight of $5000 \mathrm{~kg} / \mathrm{mol}$ and $55 \%$ of cationic $N$, $\mathrm{N}, \mathrm{N}$-trimethylaminoethylacrylate units. The concentration of stock solution was $0.1 \%$ (wt.\%).

\subsection{Apparatus}

A continuous laser diffraction instrument (Mastersizer 2000, Malvern, UK) was applied in a modified jar test procedure. Coagulation experiments were carried out on a speed-adjustive jar test with $50 \times 40 \mathrm{~mm}$ flat paddle impellers and a 1-L beaker at pre-set rotary speeds and the stirring time. Dynamic floc size was measured during the flocs growth by Mastersizer and a sample from beaker was circulated through transparent plastic tubing of $5 \mathrm{~mm}$ inner diameter by means of a peristaltic pump at a flow rate of $25 \mathrm{ml} \mathrm{min}^{-1}$ and then flowed back to the beaker. The peristaltic pump was located after the Mastersizer instrument to avoid the effects of possible aggregate breakage. Size measurements were taken every $40 \mathrm{~s}$ for the duration of the jar test. 
Table 1

The rotary speed (rpm) and its corresponding $G$ value $\left(\mathrm{s}^{-1}\right)$

\begin{tabular}{lc}
\hline Rotary speed $(\mathrm{rpm})$ & $G$ value $\left(\mathrm{s}^{-1}\right)$ \\
\hline 40 & 11.3 \\
80 & 29.1 \\
150 & 67.7 \\
200 & 99.7 \\
300 & 172.1 \\
400 & 253.4 \\
500 & 342.0 \\
\hline
\end{tabular}

\subsection{Procedure}

$800 \mathrm{ml}$ tap water was used in the jar test and the kaolin concentration in the suspension was $50 \mathrm{mg} \mathrm{l}^{-1}$. The required amounts of coagulant were pipetted into the beaker. Alum was added at the beginning of rapid mixing, while, PAM was added after $30 \mathrm{~s}$ of rapid mixing. For the determination of alum dose related to charge neutralization and sweep flocculation, the processes (the rapid mixing at $250 \mathrm{rpm}$ for $1 \mathrm{~min}$, slow mixing at $40 \mathrm{rpm}$ for $15 \mathrm{~min}$ and settling for $20 \mathrm{~min}$ ) were utilized. Furthermore, zeta potential was measured at the $30 \mathrm{~s}$ after alum dosing (Zetasizer 2000, Malvern, UK) and the turbidity of supernatant sample was determined by a turbidimeter $(2100 \mathrm{~N}$, Hach, USA). Flocs were formed by performing a series of jar test to examine the flocs structure and strength on different shear conditions. Mixing at $250 \mathrm{rpm}$ for $1 \mathrm{~min}$ was followed by floc growth phase at $40 \mathrm{rpm}, 80 \mathrm{rpm}, 150 \mathrm{rpm}, 200 \mathrm{rpm}, 300 \mathrm{rpm}$, $400 \mathrm{rpm}$, and $500 \mathrm{rpm}$ for $15 \mathrm{~min}$ respectively. Furthermore, temperature was kept at $20 \pm 3{ }^{\circ} \mathrm{C}$ on all of the experiments.

\subsection{Fractal dimension}

The fractal dimensions were determined by Mastersizer. The particles in the sample scatter light proportionally to their size and at a constant angle independent of which part of the particle is hit by the beam. Small particles scatter light at high angles, while large particles scatter at lower angles [27]. In light scattering study, the scattered intensity as a function of the magnitude of the scattering wave vector, $Q$, is measured, where,

$Q=\frac{4 \pi n \sin (\theta / 2)}{\lambda}$

where $n, \theta$, and $\lambda$ are the refractive index of the medium, the scattered angle, and the wavelength of radiation in vacuum, respectively [12]. It has been shown that for a mass fractal aggregate, which satisfies the conditions for Rayleigh-GansDebye (RGD) regime, its scattered intensity $I$ is described by the following equation [28]

$$
I \propto Q^{-D_{\mathrm{F}}}
$$

Thus, on a $\log -\log$ plot there is a liner region, with a slope of $-D_{\mathrm{F}}$.

\subsection{Floc strength calculation}

The theoretic method for calculation of floc strength was elucidated by Bache et al. [24]. $\sqrt{3} \varepsilon^{3 / 4} v^{-1 / 4}$ was cited as the turbulence acceleration when the floc size $d$ was selected to substitute for the Kolmogorow length $\eta$. The average strength per unit area at the plane of rupture was defined as $\sigma\left(\mathrm{N} \mathrm{m}^{-2}\right)$, and thus, an idealized force balance arising from opposing acceleration across weakly linked subunits of size $d$ might be written as

$\frac{1}{4} \pi d^{2} \sigma=2 \times \rho_{\mathrm{w}} \cdot \frac{\pi}{6} \eta^{3} \times \sqrt{3} \frac{\varepsilon^{3 / 4}}{v^{1 / 4}}$

where, $\rho_{\mathrm{w}}$ is the density of water $\left(\mathrm{kg} \mathrm{m}^{-3}\right), \varepsilon$ is the local rate of energy dissipation per unit mass $\left(\mathrm{m}^{2} \mathrm{~s}^{-3}\right)$. The theoretical value of floc strength could be calculated as

$\sigma=\frac{4 \sqrt{3}}{3} \frac{\rho_{\mathrm{w}} \varepsilon^{3 / 4} d}{v^{1 / 4}}\left(\mathrm{~N} \cdot \mathrm{m}^{-2}\right)$

where, $v$ is the kinematic viscosity. Furthermore, the average $\bar{\varepsilon}$ was selected to substitute for $\varepsilon, \bar{\varepsilon}$ could be calculated by

$\varepsilon=v \cdot G^{2}$

In this paper, the volumetric equivalent diameter measured by Mastersizer was used as $d(\mathrm{~m})$ and other calculation. The $G$ value of different rotary speed was listed in Table 1.

\section{Results and discussion}

\subsection{Selection of coagulant dose}

The Fig. 1 showed the variation of residual turbidity and zeta potential as a function of the alum dose. The zeta potential of original kaolin suspension was less than $-15 \mathrm{mv}$ and the turbidity was close to 50 NTU. When the alum dose was less than $0.1 \mathrm{mmol}^{-1}$, the zeta potential increased rapidly with the alum dose, on the contrary, the turbidity decreased sharply. However, as the alum dose was greater than $0.1 \mathrm{mmol}^{-1}$, the zeta potential grew up slightly, but the turbidity remained approximately constant. The charge neutralization occurred when zeta potential of particles close to zero and the sweep coagulation took place at the sufficiently high alum dose that caused precipitation of amorphous metal hydroxide. Therefore, we could consider the alum dose of $0.1 \mathrm{mmol}^{-1}$ as charge neutralization point, meanwhile $1.875 \mathrm{mmol}^{-1}$ as sweep coagulation.

For bridging, the cationic PAM was added as coagulant aid following the alum dose of $0.1 \mathrm{mmol}^{-1}$. Firstly, the stable colloidal particles was destabilized by charge neutralization of alum, and then these smaller destabilized particles agglomerated to larger aggregates by bridging of organic polymer chain absorbance. In this paper, the dose of PAM for bridging mechanism was $0.0625 \mathrm{mg} \mathrm{l}^{-1}$. 


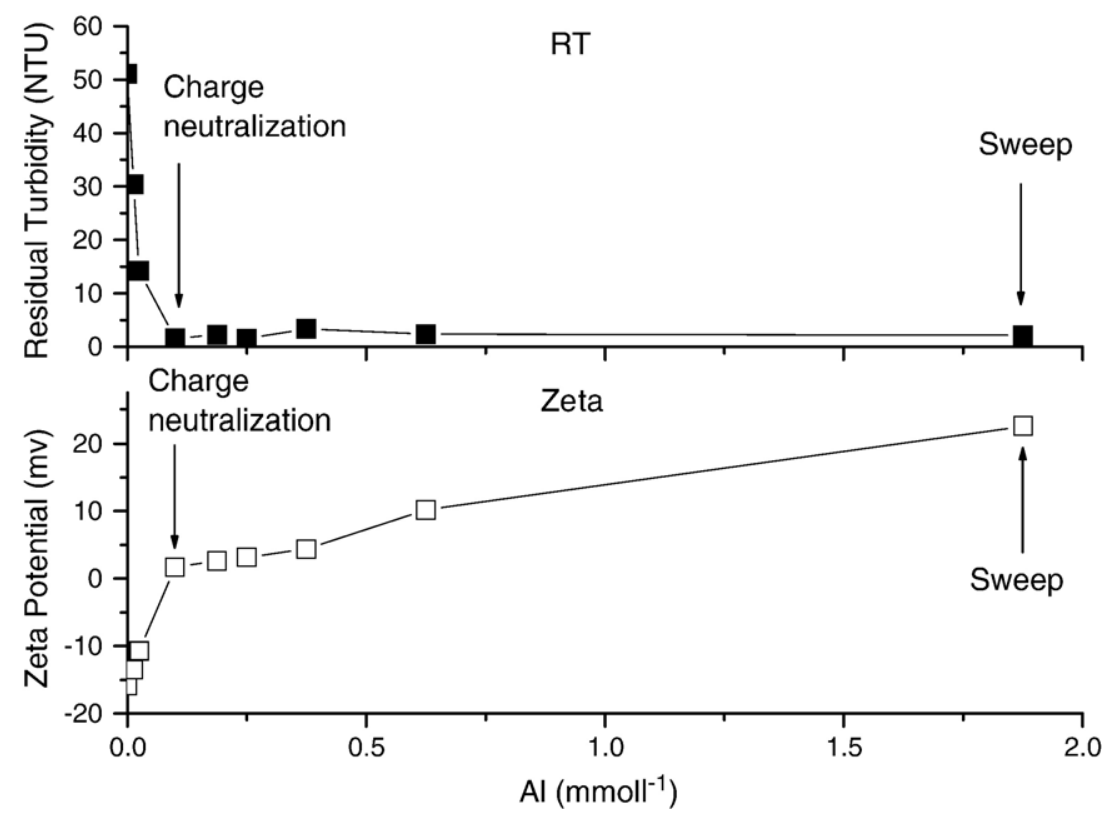

Fig. 1. The curves of turbidity and zeta potential varying with alum dose.

\subsection{Floc size, floc strength and fractal dimension}

Generally, the floc size decreases with the average hydraulic gradient $(G)$. The log-log curves of floc size decreasing with $G$ value under three mechanisms were shown in Fig. 2. Floc size was inversely proportional to the hydraulic gradient. It can be seen that all the rule of three mechanisms agree with Eq. (2), and the values of slope were also in close accord with other researchers [22,24,29]. For a given shear condition, increased floc size is a result of increased floc strength. This is because floc size is a balance between growth and breakage.
Nevertheless, the figures highlighting the distinction among the three slopes should be noted. The $\gamma$ value of charge neutralization was 0.6107 , which was the highest. Correspondingly, bridging had the lowest $\gamma$ value of 0.3674 . The result showed that the floc strength formed under charge neutralization was the weakest and there were not enough physical bonds between the flocs, and thus, the floc size decreased sharply with the shear force. While, the floc strength formed under bridging was the strongest. It can be ascribed to the strong bonds of polymer chains. For the sweep coagulation, the $\gamma$ value of 0.5618 , was between charge neutralization and bridging flocculation. Therefore, the floc

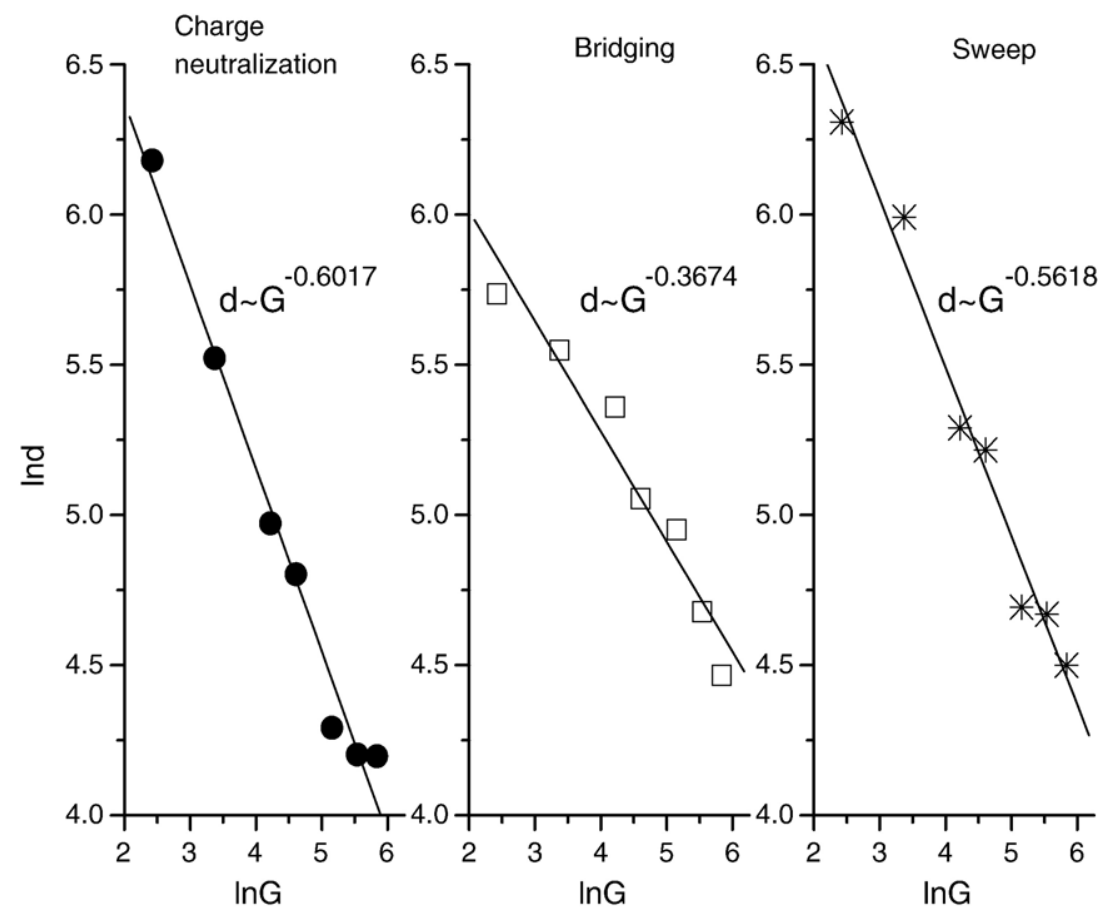

Fig. 2. The floc size $(d)$ at three coagulation mechanisms under different $G$ value $R^{2}$ was 0.98 for charge neutralization, 0.94 for sweep and 0.98 for bridging. 


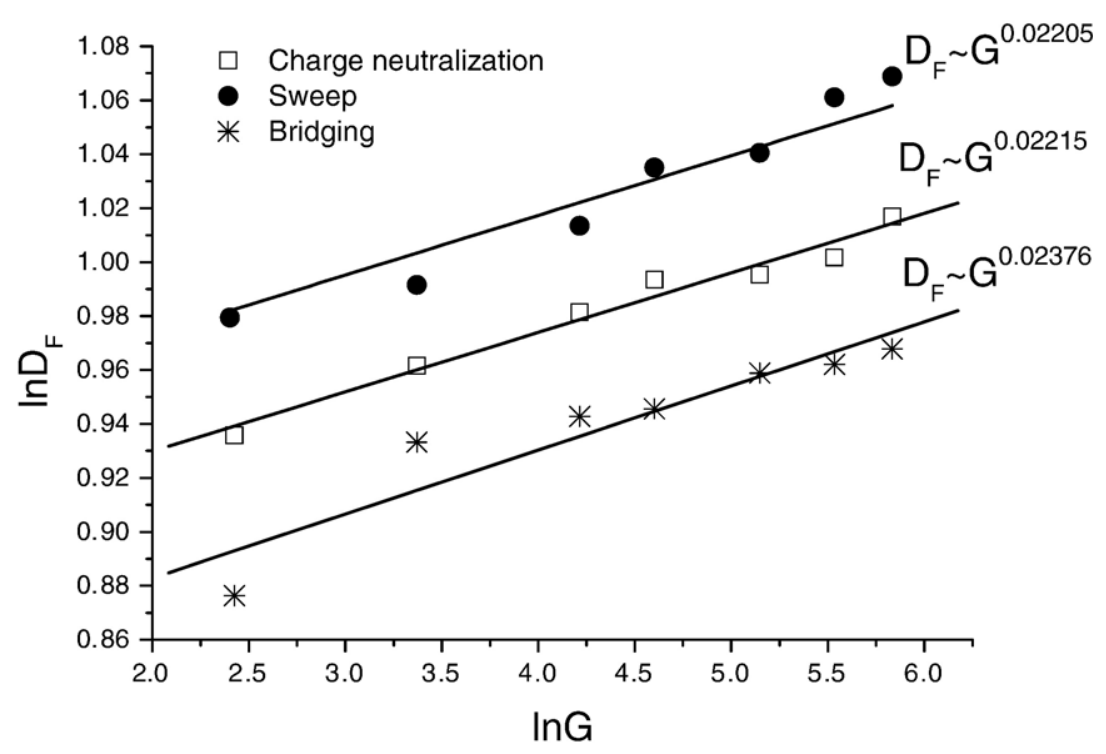

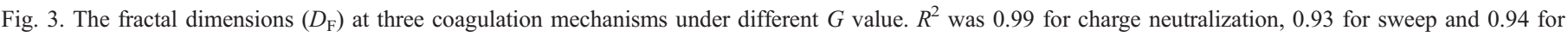
bridging.

strength was of the following hierarchy: bridging $>$ sweep $>$ charge neutralization, within the shear range investigated.

Despite the fact of floc size decreased with shear rate, the fractal dimensions increased with $G$. All of the fractal dimensions were between 2.40 and 2.91. Specifically, densely packed aggregates had a high fractal dimension, while lower fractal dimension resulted from large, highly branched and loosely bound structures. The $\log -\log$ figure of $D_{\mathrm{F}}$ and $G$ was shown in Fig. 3. As the shear with low intensity was introduced, the flocs formed with more open structure, as a result, the fractal dimensions were low. On the contrary, the more dense flocs at the high intensity shear force had higher fractal dimensions because the relatively open structure was destroyed by strong shear force and the original floc structure could not remain constant, thus the final flocs reformed with higher fractal dimension instead of branched and loosely bound structure. In summary, the shear force intensity dominated the flocs size and fractal dimensions.

Although all the fractal dimensions increased with $G$ value, the difference among three different coagulation mechanisms should be pointed out. The floc fractal dimensions under sweep mechanism were between 2.58 and 2.91, followed by charge neutralization flocs with $2.55 \sim 2.76$, and bridging flocs with $2.40 \sim 2.63$. As shown in Fig. 3, at the same hydraulic conditions, fractal dimensions of flocs formed by sweep coagulation were the highest. On the contrary, fractal dimensions of flocs by bridging flocculation were the lowest. The results showed that the fractal dimensions were affected by the complexion between flocs, which was obviously different at different coagulation mechanisms. When the sweep coagulation

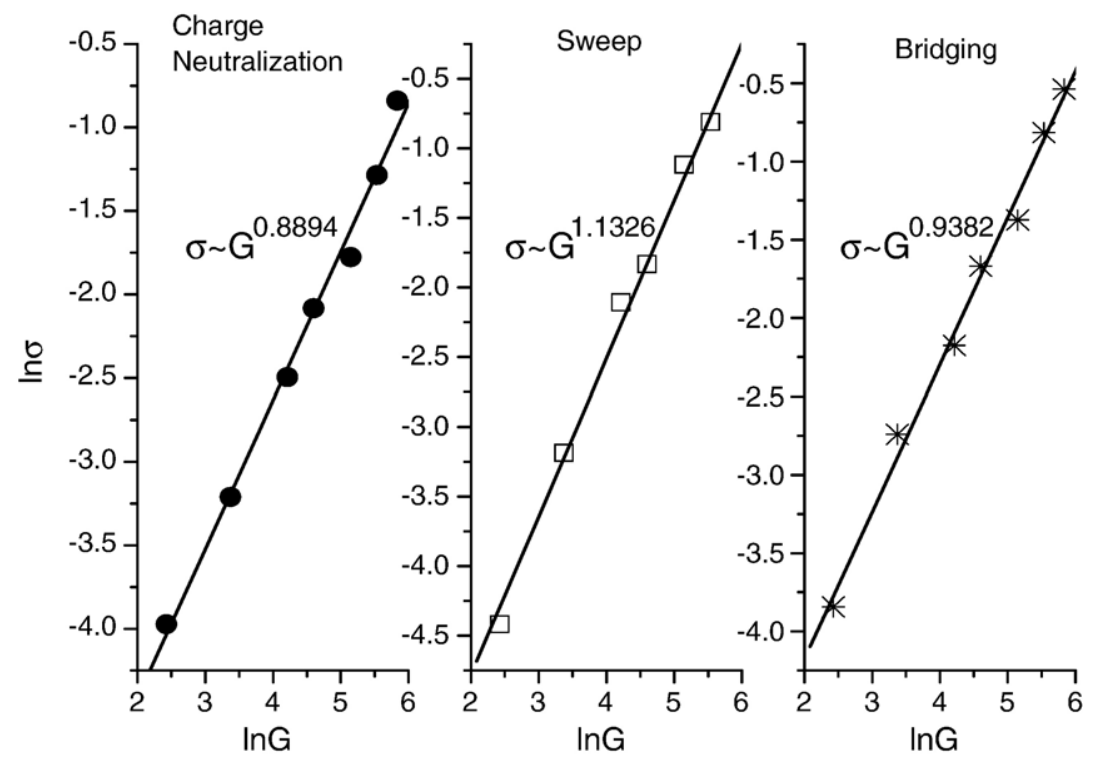

Fig. 4. The floc strength $(\sigma)$ at three coagulation mechanisms under different $G$ value. $R^{2}$ was 0.99 for all the coagulation mechanisms. 


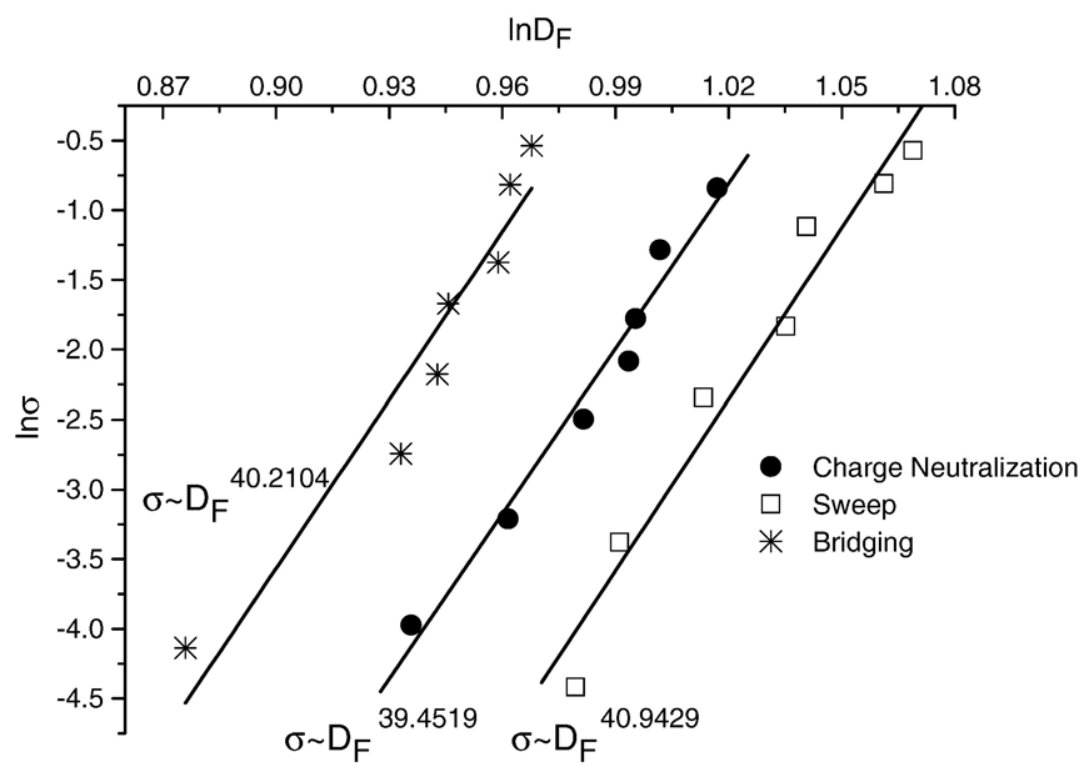

Fig. 5. The relationship between fractal dimensions $\left(D_{\mathrm{F}}\right)$ and floc strength $(\sigma) . R^{2}$ was 0.98 for charge neutralization, 0.96 for sweep and 0.92 for bridging.

dominated, the kaolin particles were enmeshed in the precipitation of amorphous aluminum hydroxide; therefore, the flocs structure was most compact. By comparing, the flocs structure was the loosest as a result of bridging flocculation, on account of the polymer molecules connecting the particles that were destabilized by alum. From Fig. 3, it was apparent that the slopes of three different coagulation mechanisms approach to the same value of 0.022 . It turned out that the variation of fractal dimensions directly proportional to $G$ value was independent of the coagulation mechanisms. Therefore, we could draw a conclusion that there was intrinsic unity for the rule of fractal dimension variation.

Floc strength calculations showed that the flocs at high $G$ value were smaller but with higher strength. The floc strength increased from 0.02 to $0.43 \mathrm{~N} \mathrm{~m}^{-2}$ under charge neutralization coagulation, and grew from 0.01 up to $0.56 \mathrm{~N} \mathrm{~m}^{-2}$ when sweep coagulation happened. However, the range of floc strength at bridging flocculation was between 0.02 and $0.58 \mathrm{~N} \mathrm{~m}^{-2}$. All the values of strength in this paper were close to the study by Bache et al. [24]. It can be seen from Fig. 4 that the relation of $G$ and floc strength on double logarithmic curves was linear. Based on different coagulation mechanisms, the slope was of the following sequence: sweep (1.1326)> bridging (0.9382)> charge neutralization (0.8894). Nevertheless, it was only a little different on these three mechanisms, and thus, obvious distinction of floc strength could not be pointed out. In conclusion, the floc strength was determined by hydraulic conditions.

The inner relationship between fractal dimensions and strength was shown in Fig. 5. All the slopes of three different mechanisms were similar and close to 40 ; therefore, there was intrinsic unity between fractal dimensions and strength, too. But, as the same fractal dimensions considered, the floc strength was of the following order: bridging $>$ charge neutralization $>$ sweep. The bridging flocculation occurred when lots of reactive groups of polymer were absorbed onto the particles, and a particlepolymer-particle aggregate was formed in which the polymer served as a bridge, therefore, the bonds of the aggregates were enough and then more loose structure with low fractal dimensions was formed on the same shear force. Compared with bridging flocculation, the floc strength by sweep coagulation was weaker at the same fractal dimensions since there was electrostatic repulsion between the particles when considering the Zeta potential was close to $20 \mathrm{mv}$, therefore, the bonds formed by aluminum hydroxide were not strong. Furthermore, floc strength of charge neutralization was moderate, as a result of the fact that the strength of physical bonds between flocs was lower than which of the chemical bonds. It was consistent with Selomulya et al. [30]. With fractal dimensions of different floc strength taken into consideration, flocs with more open structure of lower fractal dimensions would form under bridging flocculation, meanwhile, the floc size would be larger, and thus the performance of separation process was improved.

\section{Conclusion}

Floc size, structure and strength play a significant role in particles separation in water and wastewater treatment. The experimental results showed that there were fundamentally different characteristics of flocs that were generated by various coagulation mechanisms. The floc strength was of the following hierarchy: bridging $>$ charge neutralization $>$ sweep, and there was intrinsic unity between fractal dimensions and strength. At the same hydraulic conditions, fractal dimensions of flocs formed by bridging were the highest, but lowest when generated by bridging flocculation. It was also found that the floc strength was determined by hydraulic conditions.

\section{Acknowledgements}

The study was funded by the National Natural Science Foundation of China under 20477054, 20477010 and 50578155. 


\section{References}

[1] W.J. Snodgrass, M.M. Clark, C.R. O'Melia, Particle formation and growth in dilute aluminum (III) solutions, Water Research 18 (1984) 479-488.

[2] R.F. Packham, Some studies of the coagulation of dispersed clays with hydrolyzing salts, Journal of Colloid and Interface Science (1965) 20-28.

[3] B.A. Bolto, Soluble polymers in water purification, Progress in Polymer Science 20 (1995) 987-1041.

[4] M.A. Yukselen, J. Gregory, The reversibility of floc breakage, International Journal of Mineral Processing 73 (2004) 251-259.

[5] D.T. Ray, R. Hogg, Agglomerate breakage in polymer-flocculated suspensions, Journal of Colloid and Interface Science 116 (1987) $256-268$.

[6] J. Gregory, The density of particle aggregates, Water Science and Technology 36 (1997) 1-13.

[7] R.K. Chakraborti, J.F. Atkinson, J.E. Van Benschoten, Characterization of alum floc by image analysis, Environmental Science and Technology 34 (2000) 3969-3976.

[8] Q. Jiang, B.E. Logan, Fractal dimensions of aggregates determined from steady-state size distributions, Environmental Science and Technology 25 (1991) 2031-2038.

[9] C.P. Johnson, X.Y. Li, B.E. Logan, Settling velocities of fractal aggregates, Environmental Science and Technology 30 (1996) 1911-1918.

[10] D.G. Lee, J.S. Bonner, L.S. Garton, A.N.S. Ernest, R.L. Autenrieth, Modeling coagulation kinetics incorporating fractal theories: comparison with observed data, Water Research 36 (2002) 1056-1066.

[11] G.C. Bushell, Y.D. Yan, D. Woodfield, J. Raper, R. Amal, On techniques for the measurement of the mass fractal dimension of aggregates, Advances in Colloid and Interface Science 95 (2002) 1-50.

[12] R.M. Wu, D.J. Lee, T.D. Waite, J. Guan, Multilevel structure of sludge flocs, Journal of Colloid and Interface Science 252 (2002) 383-392.

[13] G. Koen, V. Willy, Image analysis to estimate the settleability and concentration of activated sludge, Water Research 31 (1997) 1126-1134.

[14] R.K. Chakraborti, K.H. Gardenr, J.F. Atkinson, J.E. Van Benschoten, Changes in fractal dimension during aggregation, Water Research 37 (2003) 873-883.

[15] P. Tang, J. Greenwood, J.A. Raper, A model to describe the settling behavior of fractal aggregates, Journal of Colloid and Interface Science 247 (2002) 210-219.

[16] M. Hermawan, G.C. Bushell, V.S.J. Craig, W.Y. Teoh, R. Amal, Floc strength characterization technique. A insight into silica aggregation, Langmuir 20 (2004) 6450-6457.
[17] D.H. Bache, C. Johnson, J.F. McGilligan, E. Rasool, A conceptual view of floc structure in the sweep floc domain, Water Science and Technology 36 (1997) 49-56.

[18] M. Boller, S. Blaser, Particles under stress, Water Science and Technology 37 (1998) 9-29.

[19] P. Jarvis, B. Jefferson, J. Gregory, S.A. Parsons, A review of floc strength and breakage, Water Research 39 (2005) 3121-3137.

[20] S.B. Francois, Strength of aluminum hydroxide flocs, Water Research 21 (1987) $1023-1030$.

[21] A.K. Yeung, R. Pelton, Micromechanics: a new approach to studying the strength and breakup of flocs, Journal of Colloid and Interface Science 184 (1996) 579-585.

[22] J. Leentvaar, M. Rebhun, Strength of ferric hydroxide flocs, Water Research 17 (1983) 895-902.

[23] P.T. Spicer, S.E. Pratsinis, Shear-induced flocculation: The evolution of floc structure and the shape of the size distribution at steady state, Water Research 30 (1996) 1049-1056.

[24] D.H. Bache, E.R. Rasool, D. Moffat, F.J. McGilligan, On the strength and character of alumino-humic flocs, Water Science and Technology 40 (1999) 81-88.

[25] P.T. Spicer, S.E. Pratsinis, J. Raper, R. Amol, G. Bushell, G. Meesters, Effect of shear schedule on particle size, density, and structure during flocculation in stirred tanks, Powder Technology 97 (1998) 26-34.

[26] S.J. Jung, R. Amal, J.A. Raper, Monitoring effects of shearing on floc structure using small-angle light scattering, Powder Technology 88 (1996) $51-54$.

[27] P. Jarvis, B. Jefferson, S.A. Parsons, Breakage, regrowth, and fractal nature of natural organic matter flocs, Environmental Science and Technology 39 (2005) 2307-2314.

[28] S. Biggs, M. Habgoog, G.J. Jameson, Yao-de Yan, Aggregate structures formed via a bridging flocculation mechanism, Chemical, Engineering Journal 80 (2000) 13-22.

[29] D.H. Bache, E.R. Rasool, Characteristics of alumino-humic flocs in relation to DAF performance, Water Science and Technology 43 (2001) 203-208.

[30] C. Selomulya, G. Bushell, R. Amal, T.D. Waite, Aggregate properties in relation to aggregation conditions under various applied shear environments, International Journal of Mineral Processing 73 (2004) 295-307. 